\title{
Mineralização de nitrogênio em solo tropical tratado com lodos de esgoto(1)
}

\author{
Rita Carla Boeira ${ }^{(2)}$, Marcos Antonio Vieira Ligo(2) e José Flávio Dynia(2)
}

\begin{abstract}
Resumo - A mineralização do N orgânico é um dos principais fatores que determinam as quantidades de lodos de esgoto (LE) a aplicar em solos. O objetivo deste trabalho foi quantificar, em laboratório, o potencial de mineralização de N orgânico num Latossolo Vermelho distroférrico, tratado com dois LE anaeróbios, um de origem estritamente urbana (Franca, SP) e outro com presença de despejos industriais (Barueri, SP). Os LE foram aplicados ao solo em doses de 1,5, 3, 6 e $12 \mathrm{~g} \mathrm{~kg}^{-1}$ (Franca) e 4, 8, 16 e $32 \mathrm{~g} \mathrm{~kg}^{-1}$ (Barueri), e o tempo de incubação foi de 15 semanas. $\mathrm{O}$ acúmulo de $\mathrm{N}$ inorgânico no solo ao final da incubação foi proporcional às quantidades de $\mathrm{N}$ orgânico adicionadas. $\mathrm{O}$ potencial de mineralização estimado pelo modelo exponencial simples foi de $24 \mathrm{mg} \mathrm{kg}^{-1}$ de $\mathrm{N}$ no solo sem lodo, e variou entre 44 e $265 \mathrm{mg} \mathrm{kg}^{-1}$ de $\mathrm{N}$ no solo tratado com os lodos. A fração de mineralização potencial do $\mathrm{N}$ orgânico dos lodos foi estimada em 31\%. A mineralização foi mais lenta no solo tratado com as duas maiores doses do LE de Barueri. Os dois lodos acidificaram o solo; o de Franca causou acidificação mais intensa que o de Barueri.
\end{abstract}

Termos para indexação: Latossolo, acidificação, $\mathrm{pH}$, condutividade elétrica.

\section{Nitrogen mineralization in a tropical soil amended with sewage sludges}

\begin{abstract}
Mineralization of organic N contained in sewage sludges is a key factor in determining the rates of these materials to be applied to agricultural soils. This work aimed at quantifying the potential of mineralization of the organic $\mathrm{N}$ in an Oxisol treated with two anaerobically-digested sewage sludges (one derived from strictly urban sewage - Franca, São Paulo State, Brazil -, and the other from a mixture of urban and industrial sewages - Barueri, São Paulo State). The sludges were applied at four rates: 1.5, 3, 6 and $12 \mathrm{~g} \mathrm{~kg}^{-1}$ (Franca), and 4, 8, 16 and $32 \mathrm{~g} \mathrm{~kg}^{-1}$ (Barueri), and the incubation period, was 15 weeks. Net inorganic $\mathrm{N}$ accumulation in the soil measured at the end of the incubation period was proportional to the rate of organic $\mathrm{N}$ applied as sludges. The potential of mineralization estimated by the single exponential model for the soil without sludge was $24 \mathrm{mg} \mathrm{kg}^{-1}$ of $\mathrm{N}$, and ranged from 44 to $265 \mathrm{mg} \mathrm{kg}^{-1}$ of $\mathrm{N}$, for the sludge-treated soil. The fraction of potential mineralization of the organic $\mathrm{N}$ contained in the sludges was estimated in $31 \%$. N mineralization was slower in the soil treated with the two higher rates of the Barueri sludge. Sewage sludges caused soil acidification; Franca sludge was more effective in this way than Barueri sludge.
\end{abstract}

Index terms: Latosol, acidification, $\mathrm{pH}$, electrical conductivity.

\section{Introdução}

Em virtude da poluição provocada por lançamento de esgotos in natura em cursos d'água, estações de tratamento de esgoto vêm sendo implementadas nas principais cidades brasileiras, obtendo-se, geralmente, considerável redução da carga orgânica dos

\footnotetext{
(1) Aceito para publicação em 9 de julho de 2002.

(2) Embrapa-Centro Nacional de Pesquisa de Monitoramento e Avaliação de Impacto Ambiental, Caixa Postal 69, CEP 13820-000 Jaguariúna, SP. E-mail:rcboeira@cnpma.embrapa.br, ligo@cnpma.embrapa.br, dynia@cnpma.embrapa.br
}

efluentes. No entanto, as estações de tratamento de esgoto defrontam-se com novo problema: a destinação a ser dada ao lodo de esgoto, resíduo que permanece após o tratamento dos esgotos. Diversas alternativas têm sido buscadas, entre elas a utilização de solos como meio de descarte para o lodo, situação em que há risco de poluição ambiental, com possibilidade de contaminação da cadeia alimentar com diversas substâncias e elementos nocivos.

Utilizado com fins agrícolas, o lodo pode atuar como condicionador de solo, por sua elevada carga orgânica ( $40 \%$ a $60 \%$ ), e como fonte de $\mathrm{N}$ para as plantas, por conter teores elevados desse nutriente 
(até 8\%), bem como possibilitar a reciclagem de outros nutrientes. Entretanto, alguns lodos podem apresentar restrições ao uso agrícola, dependendo de sua composição com metais pesados, compostos orgânicos tóxicos, ou presença de patógenos que causem risco à saúde humana ou animal. Podem, ainda, causar problemas como, por exemplo, salinização e acidificação do solo. Quando não ocorrem tais restrições, as quantidades de lodo a serem aplicadas visando à nutrição nitrogenada das culturas devem atender a dois objetivos, fundamentalmente: satisfazer as necessidades de $\mathrm{N}$ das plantas, e evitar a geração de nitrato em quantidades excessivas que venham a lixiviar no perfil do solo, colocando em risco a qualidade das águas subsuperficiais (Gangbazo et al., 1995).

O conhecimento dos atributos do lodo relacionados à degradação microbiológica do $\mathrm{N}$ orgânico pode contribuir para a previsão de seu comportamento no solo, permitindo definir parâmetros úteis ao estabelecimento das doses máximas a serem aplicadas aos solos, em função do $\mathrm{N}$ disponibilizado às plantas. $\mathrm{O} \mathrm{N}$ disponível às plantas é definido como soma do $\mathrm{N}$ na forma de nitrato $\left(\mathrm{N}-\mathrm{NO}_{3}{ }^{-}\right)$, do $\mathrm{N}$ na forma de amônio $\left(\mathrm{N}^{-\mathrm{NH}_{4}}{ }^{+}\right.$), quando não são perdidos por desnitrificação ou volatilização, respectivamente, e do $\mathrm{N}$ orgânico que é mineralizado em determinado tempo (Gilmour \& Skinner, 1999).

No estudo da dinâmica de mineralização do N orgânico de lodos aplicados a solos, tem-se adotado a premissa de que esta pode ser adequadamente descrita por uma equação cinética de primeira ordem, tal como ocorre com a mineralização do N orgânico nativo nos solos (Stanford \& Smith, 1972). Ao utilizar-se este modelo matemático, pressupõe-se que, sob certas condições ambientais, a taxa de mineralização de $\mathrm{N}$ é proporcional à quantidade de substrato mineralizável no solo. Os parâmetros obtidos com este modelo (potencial de mineralização e constante da taxa de mineralização) são úteis na definição do balanço entre as necessidades da cultura e o $\mathrm{N}$ fornecido pelo lodo, estimando-se a quantidade de $\mathrm{N}$ mineral que será liberada em determinado período de tempo, geralmente considerado como um ciclo de cultivo agrícola.

A quantidade de $\mathrm{N}$ mineralizado da matéria orgânica de lodos de esgoto é variável de acordo com o material de origem e com o processo de tratamento utilizado. De forma geral, são resíduos com estreita relação C:N, com baixo suprimento de material energético, e com material protéico de fácil degradação pelos microrganismos (Lerch et al., 1993). Estas propriedades possibilitam rápida liberação de $\mathrm{N} \mathrm{mi-}$ neral, em quantidades proporcionais às quantidades de N orgânico aplicadas (Ryan et al., 1973; Epstein et al., 1978; Gilmour \& Skinner, 1999; Rowell et al., 2001). Pode haver grande variação na fração de mineralização de lodos digeridos sob condições anaeróbias. Ryan et al. (1973) obtiveram frações de 4\% a 48\%, em 112 dias de incubação; Parker \& Sommers (1983) obtiveram valores entre $2 \%$ e $27 \%$, avaliando 13 lodos durante 112 dias. A amplitude desses resultados mostra a necessidade da quantificação da fração de mineralização de cada tipo de lodo no solo em que será aplicado.

O objetivo deste trabalho foi quantificar, em laboratório, o potencial de mineralização do $\mathrm{N}$ orgânico em um Latossolo tratado com doses de dois lodos de esgoto, um de origem estritamente urbana e outro com presença de despejos industriais.

\section{Material e Métodos}

O solo utilizado foi coletado na área experimental da Embrapa-Centro Nacional de Pesquisa de Monitoramento e Avaliação de Impacto Ambiental, situada em Jaguariúna, SP, classificado como Latossolo Vermelho distroférrico, com textura argilosa.

Os lodos de esgoto utilizados foram coletados em 1999, nas estações de tratamento de esgotos sanitários das cidades de Franca e Barueri, SP. Ambas utilizam como processo de tratamento a digestão anaeróbia do lodo ativado. A estação de Franca recebe esgotos estritamente domésticos, enquanto a de Barueri, além de esgotos domésticos, recebe também esgotos industriais. As amostras de solo $(0-20 \mathrm{~cm})$ e dos lodos foram secadas ao ar e passadas em peneira com malhas de $2 \mathrm{~mm}$. A composição química parcial do solo foi: $\mathrm{pH}, 5,8 ; \mathrm{P}, 3,5 \mathrm{mg} \mathrm{dm}{ }^{-3} ; \mathrm{K}^{+}, \mathrm{Ca}^{2+}, \mathrm{Mg}^{2+}$, $\mathrm{Al}^{3+}$ e capacidade de troca de cátions, 1,51, 27,5, 8,5, 1,0 e 73,5 $\mathrm{mmol}_{\mathrm{c}} \mathrm{dm}^{-3}$, respectivamente, determinada segundo métodos descritos por Raij \& Quaggio (1983). A composição química parcial dos lodos de Franca e Barueri é mostrada na Tabela 1. Os teores de $\mathrm{Ne} \mathrm{C}$ foram determinados utilizando-se um analisador simultâneo de $\mathrm{N}$ e Carbono. Os metais foram extraídos por digestão úmida em sistema aberto, com mistura de $\mathrm{HCl}$ e $\mathrm{HNO}_{3}$ na 
relação 3:1 (água régia) e determinados por espectrometria de emissão atômica com plasma de acoplamento indutivo.

Os tratamentos constituíram-se da testemunha (solo sem lodo), NPK (adubação mineral), 1,5, 3, 6e $12 \mathrm{~g} \mathrm{~kg}^{-1}$ de lodo de Franca e 4, 8, 16 e $32 \mathrm{~g} \mathrm{~kg}^{-1}$ de lodo de Barueri. No tratamento NPK foi feita aplicação uniforme de solução contendo $\mathrm{N}$ (50 mg kg${ }^{-1}$ de solo), P $\left(21,2 \mathrm{mg} \mathrm{kg}^{-1}\right)$ e K (26,7 $\left.\mathrm{mg} \mathrm{kg}^{-1}\right)$, utilizando-se como fontes fosfato ácido de potássio e nitrato de amônio. Os tratamentos foram denominados de acordo com as quantidades de $\mathrm{N}$ orgânico adicionadas ao solo (mg de $\mathrm{N}$ orgânico por $\mathrm{kg}$ de solo) e com o tipo de lodo (F: Franca; B: Barueri): 63 F, 122 F, 244 F e 486 F; 106 B, 201 B, 400 B e 796 B. As quantidades de N orgânico aplicadas ao solo foram calculadas subtraindo-se o $\mathrm{N}$ mineral $\left[\mathrm{N}-\left(\mathrm{NO}_{3}{ }^{-}+\mathrm{NO}_{2}{ }^{-}\right)+\mathrm{N}-\mathrm{NH}_{4}{ }^{+}\right]$, extraído na época zero dia, do $\mathrm{N}$ total ( $\mathrm{N}$ orgânico $+\mathrm{N}$ mineral) aplicado via lodo. As épocas de avaliação foram: 0, 7, 14, 21, 28, 42, 63, 84 e 105 dias. Os tratamentos foram organizados em arranjo fatorial 10x9 (dez doses de lodo e nove épocas de avaliação), em delineamento experimental em blocos casualizados, com três repetições.

Utilizou-se o teste de Tukey para comparação de médias, quando indicado pela análise de variância, e análise de correlação e de regressão linear dos dados, a 5\% de probabilidade. As parcelas experimentais constituíram-se de vasos de plástico com capacidade para $100 \mathrm{~g}$ da mistura lodo+solo. A incubação foi conduzida em sala mantida à temperatura média de $24^{\circ} \mathrm{C}$ e umidade relativa média do ar de $60 \%$. A umidade do solo nos vasos foi controlada por pesagem, e mantida à capacidade de campo por meio de regas periódicas, com a água ocupando aproximadamente $60 \%$ do volume total de poros (-3 MPa).

Em cada época, as avaliações foram feitas em diferentes unidades experimentais (processo destrutivo), após homogeneização do solo. Em todas as épocas de avaliação, foram determinados: $\mathrm{N}-\left(\mathrm{NO}_{3}{ }^{-}+\mathrm{NO}_{2}^{-}\right)$e $\mathrm{N}-\mathrm{NH}_{4}{ }^{+}$por destilação a vapor (Tedesco et al., 1995) e pH (solo:água 1:2,5); nas épocas 0 dia e 63 dias, foi determinado $\mathrm{C}$ orgânico (Tedesco et al., 1995); nas épocas 0, 14, 28 e 63 dias, foi determinada a condutividade elétrica (solo:água 1:5) e na época zero dia, foi avaliado o $\mathrm{N}$ total nos tratamentos
(Tedesco et al., 1995). As determinações de $\mathrm{N}-\left(\mathrm{NO}_{3}{ }^{-}+\mathrm{NO}_{2}{ }^{-}\right)$e $\mathrm{N}-\mathrm{NH}_{4}{ }^{+}$foram feitas em duas repetições, devido a limitações operacionais; verificando-se diferenças superiores a $20 \%$ entre elas, a terceira repetição foi analisada. A variação líquida de $\mathrm{N}$ mineral nos tratamentos foi calculada em cada época de avaliação, a partir de sete dias, obtendo-se os valores de $\mathrm{N}$ mineral acumulado (Nm). Esses dados foram utilizados na avaliação da dinâmica da mineralização do $\mathrm{N}$ orgânico nos tratamentos (sistemas lodo-solo), utilizando-se o modelo proposto por Stanford \& Smith (1972). Este modelo é descrito por uma equação exponencial simples, assumindo-se uma reação cinética de primeira ordem: $\mathrm{Nm}=\mathrm{No}\left(1-\mathrm{e}^{-\mathrm{kt}}\right)$. Os parâmetros No (N orgânico potencialmente mineralizável no sistema lodosolo; $\mathrm{mg} \mathrm{kg}^{-1}$ ) e $\mathrm{k}$ (constante de primeira ordem da taxa de mineralização de $\mathrm{N}$ orgânico; dia $^{-1}$ ) foram estimados por análise de regressão não-linear (Smith et al., 1980); $\mathrm{t}$ refere-se ao tempo (dias). A fração de mineralização do $\mathrm{N}$ orgânico dos lodos (FM) foi calculada utilizando-se a fórmula:

FM $(\%)=[\mathrm{Nm}$ (tratamento) $-\mathrm{Nm}$ (testemunha) $] \times 100 /$ ( $\mathrm{N}$ orgânico aplicado via lodo),

onde: $\mathrm{Nm}$ (tratamento) e $\mathrm{Nm}$ (testemunha) referem-se às quantidades de $\mathrm{N}$ mineral acumuladas em 105 dias de incubação, considerando-se que o $\mathrm{N}$ nativo no solo tenha pequena contribuição sobre o potencial de mineralização de um sistema lodo-solo (Sikora \& Yakovchenko, 1996). A FM potencial dos lodos foi estimada pela fórmula: FM $(\%)=[$ No (tratamento) - No (testemunha) $] \times 100 /$ ( $\mathrm{N}$ orgânico aplicado via lodo).

\section{Resultados e Discussão}

$\mathrm{O} \mathrm{N}-\mathrm{NH}_{4}{ }^{+}$foi a forma predominante de $\mathrm{N}$ mineral nos sistemas lodo-solo nas primeiras semanas, com exceção dos tratamentos $63 \mathrm{Fe} 106$ B. De modo geral, os decréscimos de N-NH${ }_{4}^{+}$ao longo do tempo foram acompanhados de aumentos correspondentes de $\mathrm{N}-\left(\mathrm{NO}_{3}{ }^{-}+\mathrm{NO}_{2}^{-}\right)$, obtendo-se correlação

Tabela 1. Composição química parcial de lodos de esgoto coletados em 1999, nas estações de tratamento de esgoto de Franca, SP e de Barueri, SP.

\begin{tabular}{|c|c|c|c|c|c|c|c|c|c|c|c|c|c|}
\hline Lodo & $\mathrm{pH}^{(1)}$ & $\begin{array}{l}\text { Umidade } \\
\text { (base seca) }\end{array}$ & $\mathrm{N}$ total & $\mathrm{C}$ orgânico ${ }^{(2)}$ & $\mathrm{Ca}$ & $\mathrm{Mg}$ & K & $\mathrm{Cr}$ & $\mathrm{Ni}$ & $\mathrm{Cu}$ & $\mathrm{Zn}$ & $\mathrm{Cd}$ & $\mathrm{Pb}$ \\
\hline & & (5) & & $\left(\mathrm{g} \mathrm{kg}^{-1}\right)$ & ----- & & ---- & & 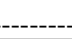 & ---- & $\mathrm{ng} \mathrm{kg}^{-1}$ & ----. & \\
\hline Franca & 6,3 & 172 & 46,0 & 308 & 13,1 & 1,7 & 0,8 & 308 & 33,9 & 153 & 744 & 2,56 & 72,6 \\
\hline Barueri & 6,5 & 96 & 26,8 & 206 & 21,5 & 2,3 & 1,4 & 418 & 289,3 & 738 & 1.765 & 8,76 & 160,5 \\
\hline
\end{tabular}

${ }^{(1)}$ Relação lodo:água 1:2,5. (2)Walkley Black. 
significativa entre essas variáveis $(r=-0,43)$, evidenciando a ocorrência da nitrificação desde o início da incubação (Figura 1). Nas doses menores (63 F e $106 \mathrm{~B}$ ), o teor de nitrato estabilizou-se a partir de 42 dias. No entanto, a nitrificação líquida nos tratamentos 486 F, 400 B e 796 B intensificou-se mais tarde (após 42 dias) em relação aos demais tratamentos. Nesses tratamentos, até 42 dias, houve permanência no solo de $\mathrm{N}$ inorgânico composto em grande parte pela forma amoniacal. Segundo Smith et al. (1998), excesso de N sob forma amoniacal no solo pode inibir o processo de nitrificação.
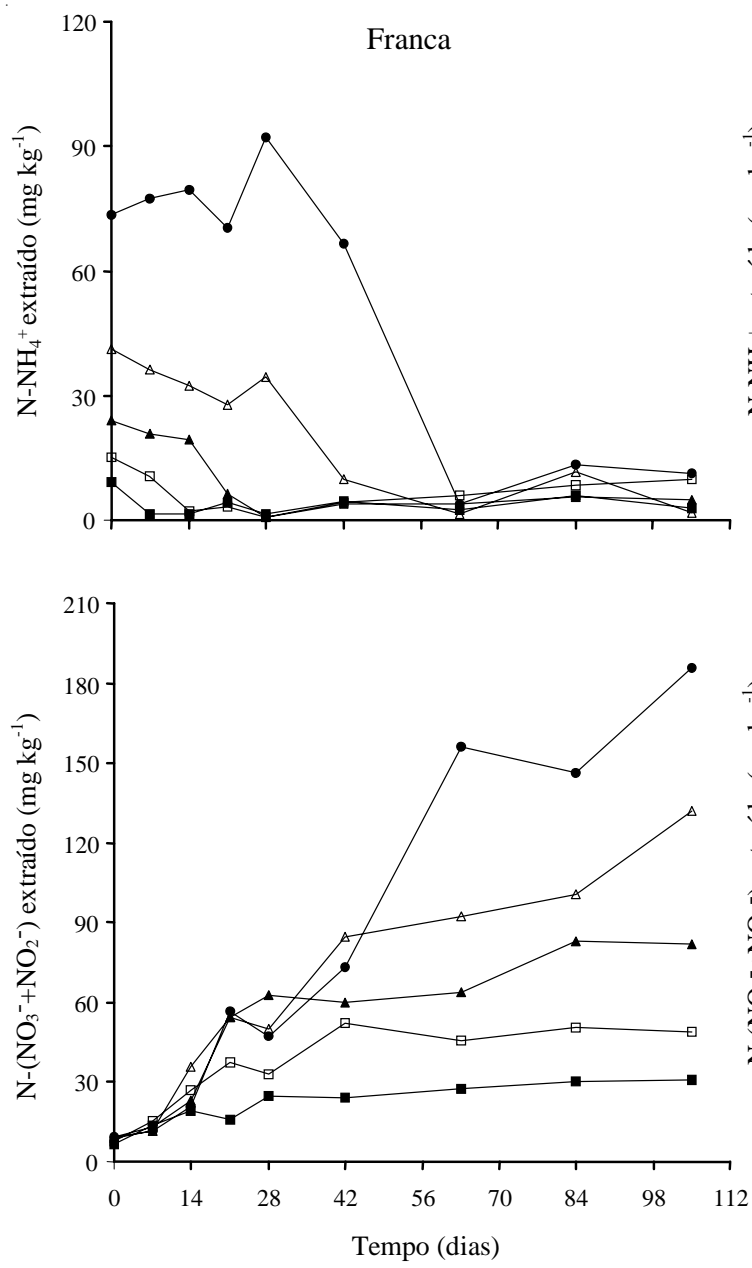

$\mathrm{O}$ acúmulo de $\mathrm{N}$ inorgânico $(\mathrm{Nm})$ durante a incubação esteve associado às quantidades de $\mathrm{N}$ orgânico adicionadas ao solo $(\mathrm{r}=0,41)$, obtendo-se regressão linear significativa aos 105 dias $\left(r^{2}=0,93\right)$. A velocidade de mineralização foi maior no início da incubação e decresceu com o tempo (Figura 2). Comportamentos semelhantes com lodos de esgoto foram constatados por Parker \& Sommers (1983), Lindemann \& Cardenas (1984) e Banerjee et al. (1997), em razão da decomposição inicial de formas nitrogenadas mais lábeis, com posterior predominância de formas recalcitrantes.
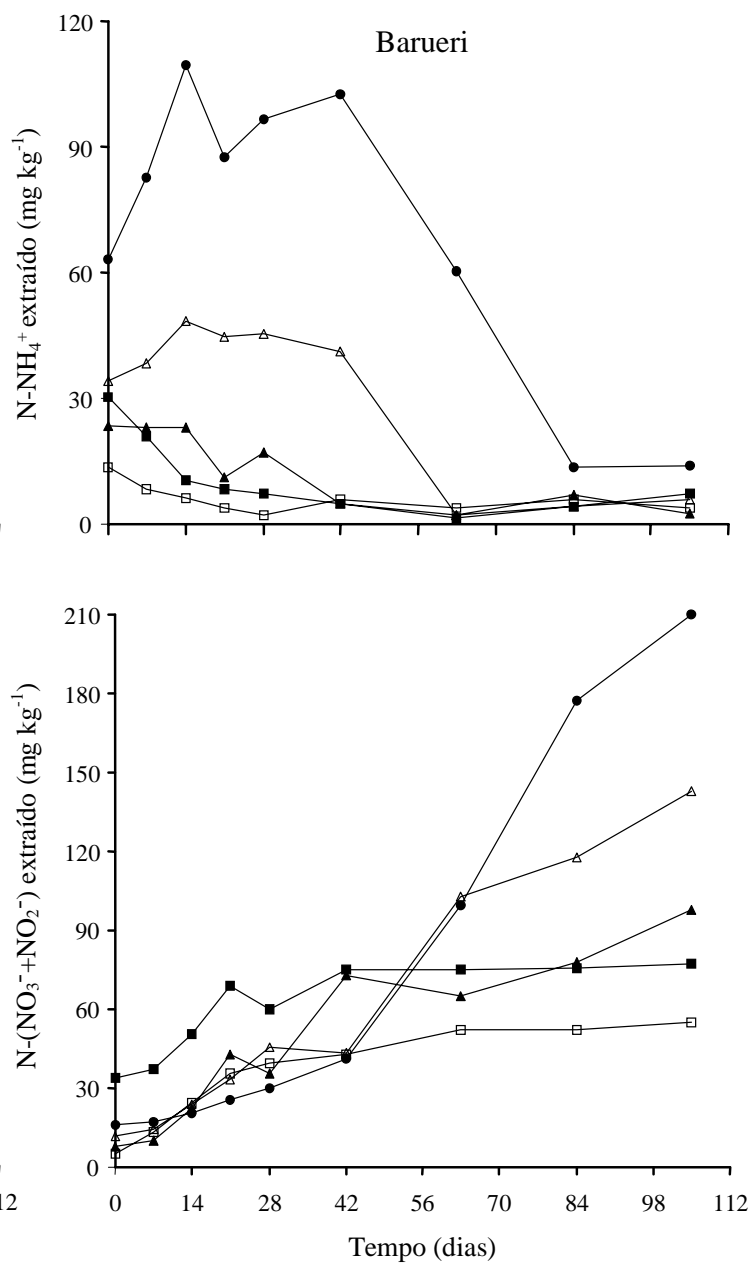

Figura 1. Teores de N-NH${ }_{4}^{+}$e de $\mathrm{N}-\left(\mathrm{NO}_{3}{ }^{+}+\mathrm{NO}_{2}{ }^{-}\right)$em Latossolo incubado durante 105 dias com lodos de esgoto coletados em 1999, nas estações de tratamento de esgoto de Franca, SP (ロ: testemunha; $\square: 63 ; \mathbf{\Lambda}: 122 ; \Delta: 244 \mathrm{e} \bullet: 486 \mathrm{mg}$ de N orgânico kg-1 de solo) e de Barueri, SP (匹: NPK; $\square: 106$; $\mathbf{\Delta}: 201 ; \Delta: 400$ e $\bullet: 796$ mg de N orgânico kg-1 de solo). 
Durante o período experimental, as quantidades de $\mathrm{N}$ mineral acumulado $(\mathrm{Nm})$ foram crescentes nos tratamentos, observando-se rápida estabilização na testemunha, NPK, 63 F e 106 B. Estes diferentes comportamentos do solo quando tratado com várias doses de lodo estão representados nos parâmetros obtidos pelo ajuste dos dados ao modelo de Stanford \& Smith (1972) (Tabela 2). Os valores estimados de No foram superiores aos do $\mathrm{N}$ mineral acumula- do em 105 dias em todos os tratamentos. Banerjee et al. (1997) e Aquino Neto (1998) também observaram essa tendência em incubações prolongadas, indicando que ainda perdura uma fração do $\mathrm{N}$ orgânico disponível a uma posterior mineralização. Cerca de $80 \%$ do $\mathrm{N}$ orgânico potencialmente mineralizável nos sistemas lodo-solo foram mineralizados aos 105 dias, exceto nos tratamentos 400 B e 796 B, que apresentaram apenas $57 \%$ de
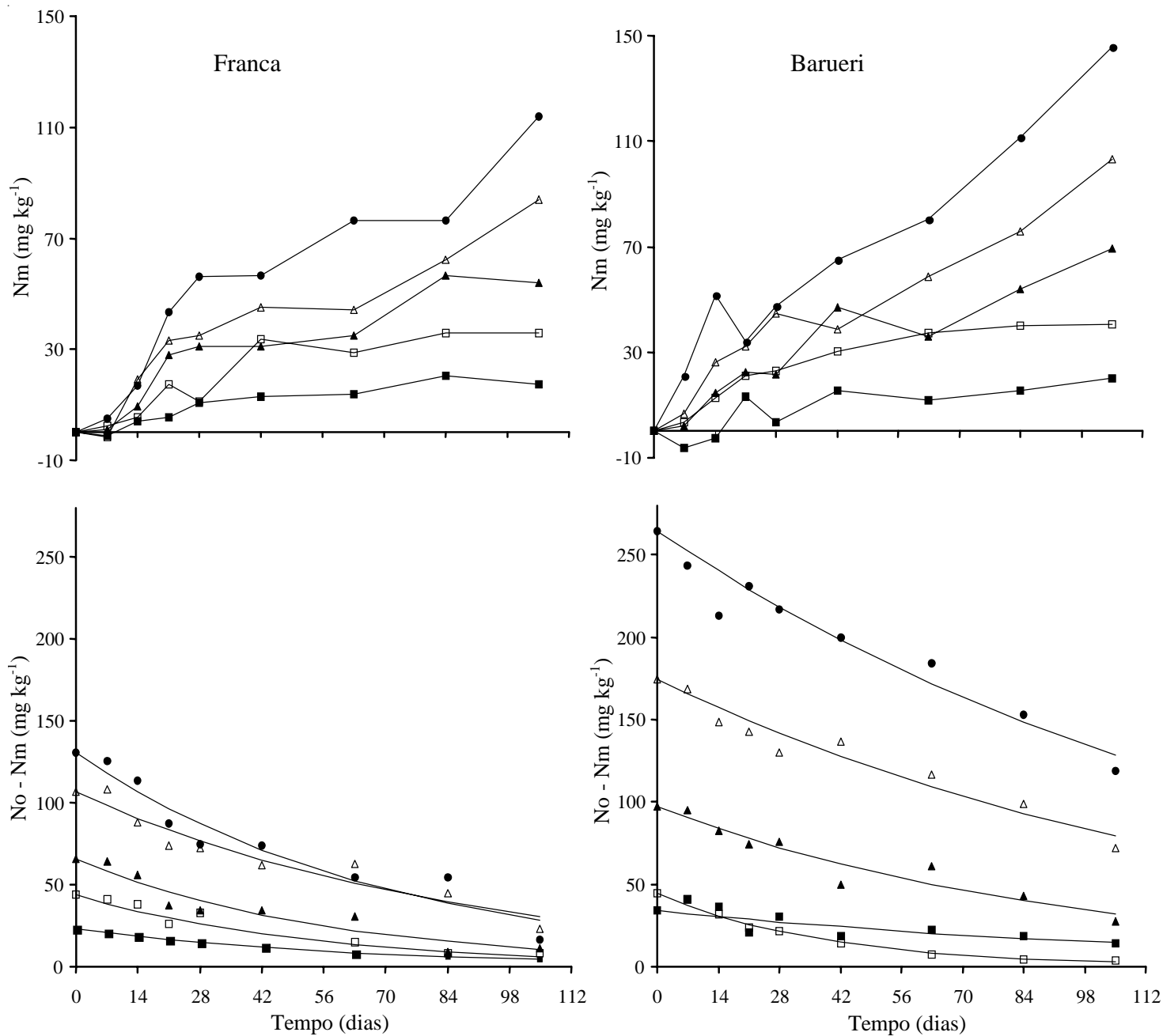

Figura 2. Variação líquida de $\mathrm{N}$ inorgânico $(\mathrm{Nm})$ e quantidade de $\mathrm{N}$ potencialmente mineralizável disponível à mineralização (No - Nm), com ajuste dos dados ao modelo exponencial simples (Stanford \& Smith, 1972) em Latossolo incubado durante 105 dias com lodos de esgoto coletados em 1999, nas estações de tratamento de esgoto de Franca, SP

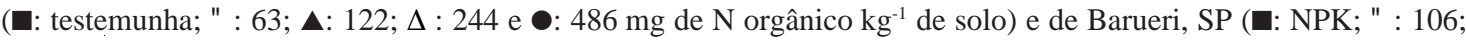
A: $201 ; \Delta: 400$ e $\mathbf{0}: 796$ mg de $\mathrm{N}$ orgânico $\mathrm{kg}^{-1}$ de solo). 
mineralização em relação a No. Na testemunha, $18 \%$ do $\mathrm{N}$ potencialmente mineralizável foi extraído entre 7 e 14 dias, $27 \%$ entre 14 e 28 dias, e, a partir daí, a velocidade de mineralização diminuiu, estimando-se que, ao final de 105 dias, tenha ocorrido $68 \%$ de mineralização. O No estimado nesse solo, $23,5 \mathrm{mg} \mathrm{kg}^{-1}$, foi pelo menos cinco vezes menor que o valor de No estimado em solos do Estado do Rio Grande do Sul, RS, por Camargo et al. (1997). Estas diferenças podem ser inerentes ao solo, que conteria formas nitrogenadas relativamente resistentes à decomposição microbiológica em relação àqueles solos, ou podem ser devidas a um meio biologicamente menos ativo, em razão de variáveis que atuam no processo, como, por exemplo, a temperatura, que no presente trabalho foi mantida a $24^{\circ} \mathrm{C}$, e naquele, a $35^{\circ} \mathrm{C}$.

A constante de mineralização $\mathrm{k}$ também foi inferior neste solo em relação aos do RS, denotando fornecimento mais lento de $\mathrm{N}$ mineral à solução do solo (1,6\% do N mineralizável presente no solo, por dia), nas condições experimentais. Silva et al. (1999) também observaram baixa disponibilidade de $\mathrm{N}$ mine- ral em Latossolos, após 70 dias de incubação e sob temperaturas variando entre $21^{\circ} \mathrm{Ce} 29^{\circ} \mathrm{C}$. Os valores de $\mathrm{k}$ obtidos nos sistemas lodo-solo variaram de 0,007 a 0,026 dia ${ }^{-1}$, a maioria dentro da faixa obtida por Parker \& Sommers (1983) para 24 lodos. A partir do valor de $\mathrm{k}$, foi calculada a meia-vida do $\mathrm{N}$ orgânico em cada sistema lodo-solo (tempo necessário para a degradação de 50\% do N orgânico). O modelo indicou velocidade de mineralização distinta entre tratamentos, com meias-vidas variando entre $26 \mathrm{e}$ 100 dias. Na relação [No - Nm] x tempo observa-se este comportamento, com mineralização mais lenta do lodo de Barueri quando aplicado em doses elevadas (400 B e 796 B), enquanto as doses $244 \mathrm{~F}$, 486 F e 201 B mostram tendência de estabilização aos 105 dias (Figura 2).

A fração de mineralização (FM) dos lodos em 105 dias de incubação variou de 16\% a 32\% (média de $25 \%$ ), sem diferenças significativas entre tratamentos (Tabela 2). Doses crescentes de lodo aplicadas ao solo também não afetaram a FM obtida por Epstein et al. (1978). Estimando-se a FM potencial com os dados do modelo, obtém-se valores entre

Tabela 2. Doses de lodo, de $\mathrm{N}$ total e de $\mathrm{N}$ mineral aplicados nos tratamentos, variação líquida observada de $\mathrm{N}$ inorgânico (Nm), médias observadas da fração de mineralização (FM) com respectivos intervalos de confiança (IC) e parâmetros das equações de ajuste dos dados ao modelo exponencial simples (Stanford \& Smith, 1972), em Latossolo incubado durante 105 dias, com lodos de esgoto coletados em 1999, nas estações de tratamento de esgoto de Franca, SP e de Barueri, SP.

\begin{tabular}{|c|c|c|c|c|c|c|c|c|c|c|}
\hline \multirow[t]{2}{*}{ Tratamento $^{(1)}$} & \multicolumn{3}{|c|}{ Doses aplicadas } & \multirow[t]{2}{*}{$\mathrm{Nm}$} & \multicolumn{2}{|c|}{ FM do lodo ${ }^{(2)}$} & \multicolumn{4}{|c|}{$\mathrm{Nm}=\mathrm{No}\left(1-\mathrm{e}^{-\mathrm{kt}}\right)$} \\
\hline & $\begin{array}{c}\text { Lodo } \\
\left(\mathrm{g} \mathrm{kg}^{-1}\right)\end{array}$ & $\mathrm{N}$ total & $\begin{array}{l}\mathrm{N} \text { mineral } \\
\left(\mathrm{mg} \mathrm{kg}^{-1}\right)-\end{array}$ & & $\begin{array}{l}\text { Médias } \\
-\end{array}$ & $\begin{array}{l}\text { IC 95\% } \\
\%) \text { - }\end{array}$ & $\begin{array}{c}\mathrm{No}^{(3)} \\
\left(\mathrm{mg} \mathrm{kg}^{-1}\right)\end{array}$ & $\begin{array}{c}\mathrm{k}^{(4)} \\
\left(\mathrm{dia}^{-1}\right)\end{array}$ & $\begin{array}{l}\text { Meia-vida }^{(5)} \\
\text { (dia) }\end{array}$ & $\begin{array}{l}\mathrm{R}^{2(6)} \\
(\%)\end{array}$ \\
\hline NPK & - & $50^{(7)}$ & 47 & 20 & - & - & 34,0 & 0,0081 & 86 & 53,0 \\
\hline Test & - & 0 & 0 & 16 & - & - & 23,5 & 0,0164 & 42 & 83,0 \\
\hline \multicolumn{11}{|c|}{ Franca } \\
\hline 63 & 1,5 & 69 & 6 & 36 & 32 & $(21-42)$ & 43,9 & 0,0189 & 37 & 85,0 \\
\hline 122 & 3,0 & 138 & 16 & 54 & 31 & $(20-42)$ & 65,5 & 0,0173 & 40 & 82,0 \\
\hline 244 & 6,0 & 276 & 32 & 84 & 28 & $(17-39)$ & 107,1 & 0,0119 & 58 & 86,0 \\
\hline 486 & 12,0 & 552 & 66 & 114 & 20 & $(9-31)$ & 131,0 & 0,0145 & 48 & 85,0 \\
\hline \multicolumn{11}{|c|}{ Barueri } \\
\hline 106 & 4,0 & 107 & 1 & 40 & 23 & $(12-34)$ & 44,6 & 0,0265 & 26 & 91,0 \\
\hline 201 & 8,0 & 214 & 14 & 69 & 26 & $(16-37)$ & 97,1 & 0,0105 & 66 & 87,0 \\
\hline 400 & 16,0 & 429 & 28 & 103 & 22 & $(11-33)$ & 174,8 & 0,0075 & 92 & 91,0 \\
\hline 796 & 32,0 & 858 & 62 & 146 & 16 & $(6-27)$ & 264,6 & 0,0069 & 100 & 87,0 \\
\hline
\end{tabular}

${ }^{(1)}$ Valores em mg de $\mathrm{N}$ orgânico $\mathrm{kg}^{-1}$ de solo. ${ }^{(2)} \mathrm{Não}$ houve diferenças significativas pelo teste de Tukey a 5\% de probabilidade. ${ }^{(3)} \mathrm{N}$ potencialmente mineralizável. ${ }^{(4)}$ Constante da taxa de mineralização. ${ }^{(5)}$ Tempo necessário para degradação de $50 \%$ do $\mathrm{N}$ orgânico do solo. ${ }^{(6)}$ Coeficiente de determinação. ${ }^{(7)} \mathrm{N}$ inorgânico adicionado. 
$20 \%$ e $38 \%$ (média de $31 \%$ ). A FM média estimada pelo modelo foi superior à FM média calculada aos 105 dias em razão dos valores de No estimados terem sido superiores às quantidades acumuladas de $\mathrm{N}$ mineral durante a incubação. A FM estimada situou-se dentro dos limites dos intervalos de confiança obtidos com relação a este parâmetro nos tratamentos, exceto em 400 B e 796 B.

$\mathrm{Na}$ avaliação da qualidade do solo, tem-se buscado identificar indicadores que sejam sensíveis às mudanças no manejo do solo. Entre eles, o pH, para avaliar a acidez, e a condutividade elétrica, como estimativa do teor de sais ou capacidade de fornecimento de nutrientes. Além de afetar a disponibilidade de nutrientes e de metais pesados às plantas, essas propriedades podem ser utilizadas como indicadores em relação ao monitoramento de nitrato a campo (Patriquin et al., 1993). A condutividade elétrica também pode afetar, indiretamente, a mineralização da matéria orgânica (McClung \& Frankenberger, 1985). O pH diminuiu no tratamento testemunha após a incubação (Figura 3), resultado também observado por Silva et al. (1999) em incubações com sete solos brasileiros.

Considerando-se todos os tratamentos e as diversas épocas, houve correlações significativas entre os valores medidos de $\mathrm{pH}$ e as quantidades extraí- das de $\mathrm{N}-\left(\mathrm{NO}_{3}{ }^{-}+\mathrm{NO}_{2}{ }^{-}\right)$e de $\mathrm{N}-\mathrm{NH}_{4}{ }^{+}(\mathrm{r}=-0,79$ e 0,54 , respectivamente). Aos 105 dias, houve decréscimos lineares no $\mathrm{pH}$ com o aumento das doses aplicadas dos lodos de Franca $\left(r^{2}=0,96\right)$ ou de Barueri $\left(r^{2}=0,87\right)$; na comparação conjunta dos dados dos dois lodos, foram significativamente mais acidificantes as doses 244 F e 486 F. Diferentemente desses resultados, Sloan \& Basta (1995) constataram aumentos lineares no $\mathrm{pH}$ com aplicação de doses crescentes de lodo anaeróbio não calado em solos ácidos. Comparando-se doses semelhantes dos dois lodos, verificou-se que a acidificação do solo nos tratamentos com o lodo de Franca foi significativamente maior do que nos tratamentos com o lodo de Barueri. Não houve diferenças significativas entre o valor inicial de condutividade elétrica e o valor medido aos 63 dias, em cada tratamento, mostrando que este indicador não foi adequado na avaliação da decomposição da carga orgânica no solo tratado com lodo. Aos 63 dias, pôde-se observar um efeito significativo das doses aplicadas de lodo sobre esta variável (Tabela 3). Rodgers \& Anderson (1995) também constataram maiores concentrações de sais com aplicação de lodo, avaliando doses crescentes até $333 \mathrm{Mg} \mathrm{ha}^{-1}$.

Outras propriedades do solo também foram avaliadas com o objetivo de verificar sua sensibilidade
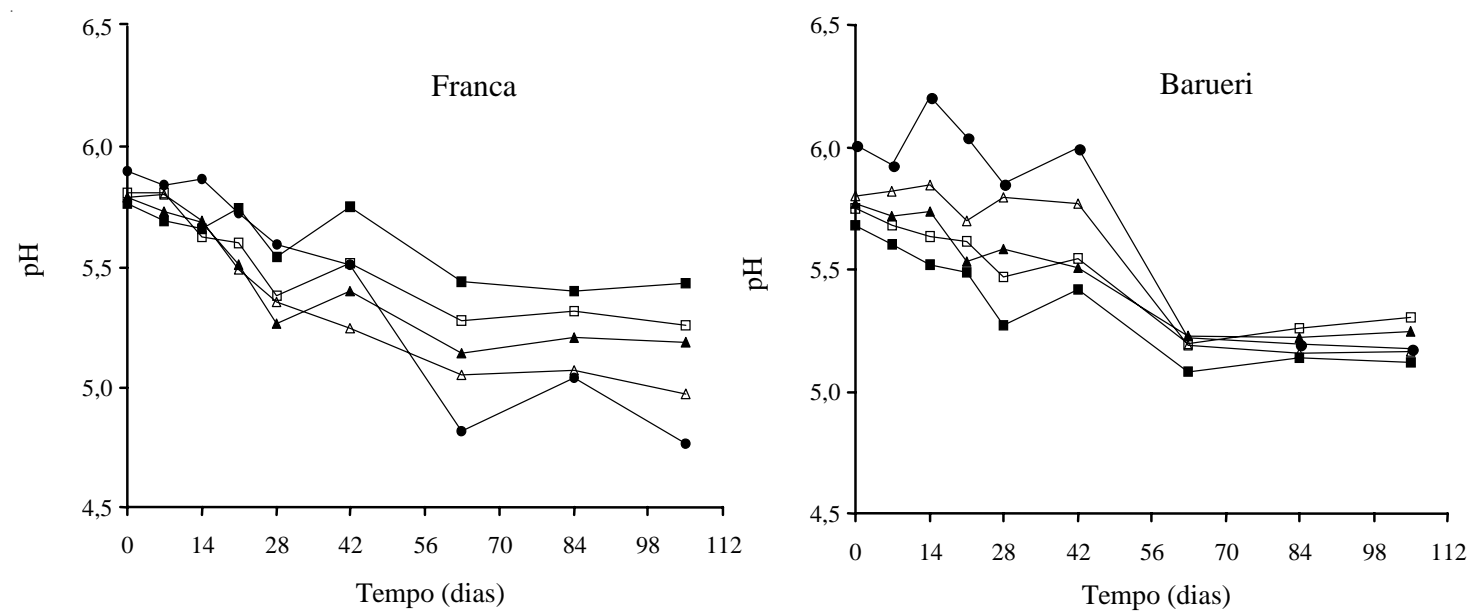

Figura 3. Valores de pH em Latossolo incubado durante 105 dias com lodos de esgoto coletados em 1999, nas estações

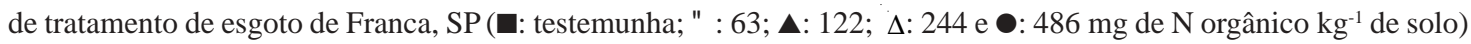
e de Barueri, SP (ם: NPK; $\square: 106 ; \boldsymbol{\Delta}: 201: \Delta: 400$ e ๑: 796 mg de N orgânico $\mathrm{kg}^{-1}$ de solo). 
Tabela 3. Teores de $\mathrm{N}$ total, $\mathrm{C}$ orgânico e condutividade elétrica em Latossolo incubado com lodos de esgoto coletados em 1999, nas estações de tratamento de esgoto de Franca, SP e de Barueri, $\mathrm{SP}^{(1)}$.

\begin{tabular}{|c|c|c|c|c|c|c|c|}
\hline \multirow[t]{3}{*}{ Tratamento $^{(2)}$} & $\mathrm{N}$ total & \multicolumn{2}{|c|}{ C orgânico } & \multicolumn{4}{|c|}{ Condutividade elétrica } \\
\hline & \multirow{2}{*}{0 dias } & 0 dias & 63 dias & 0 dias & 14 dias & 28 dias & 63 dias \\
\hline & & $-\left(\mathrm{g} \mathrm{kg}^{-1}\right)$ & & -------- & \multicolumn{2}{|c|}{$\left(\mathrm{S} \mathrm{m}^{-1}\right)$} & \\
\hline NPK & $1,26 \mathrm{a}$ & $17,08 \mathrm{a}$ & $16,42 \mathrm{ab}$ & $0,009 \mathrm{bc}$ & $0,012 \mathrm{abc}$ & $0,014 \mathrm{ab}$ & $0,016 \mathrm{c}$ \\
\hline Test & $1,22 \mathrm{a}$ & $17,44 \mathrm{a}$ & $15,69 \mathrm{a}$ & $0,005 \mathrm{a}$ & $0,006 \mathrm{a}$ & $0,006 \mathrm{a}$ & $0,008 \mathrm{a}$ \\
\hline \multicolumn{8}{|c|}{ Franca } \\
\hline 63 & $1,25 \mathrm{a}$ & $17,28 \mathrm{a}$ & $15,66 \mathrm{a}$ & $0,005 \mathrm{ab}$ & $0,009 \mathrm{ab}$ & $0,009 \mathrm{ab}$ & $0,011 b$ \\
\hline 122 & $1,45 b$ & $18,33 \mathrm{ab}$ & $16,50 \mathrm{ab}$ & $0,007 \mathrm{ab}$ & $0,011 \mathrm{abc}$ & $0,013 \mathrm{ab}$ & $0,018 \mathrm{~d}$ \\
\hline 244 & $1,32 \mathrm{ab}$ & $19,00 b c$ & $17,50 \mathrm{~b}$ & $0,010 \mathrm{bc}$ & $0,015 \mathrm{abc}$ & $0,019 b c$ & $0,024 \mathrm{e}$ \\
\hline 486 & $1,71 \mathrm{c}$ & $20,49 b c$ & $19,98 \mathrm{c}$ & $0,014 \mathrm{de}$ & $0,016 \mathrm{abc}$ & $0,022 \mathrm{bcd}$ & $0,037 \mathrm{f}$ \\
\hline \multicolumn{8}{|c|}{ Barueri } \\
\hline 106 & $1,32 \mathrm{ab}$ & $17,78 \mathrm{a}$ & $16,41 \mathrm{ab}$ & $0,008 b c$ & $0,011 b c$ & $0,013 \mathrm{ab}$ & $0,014 \mathrm{c}$ \\
\hline 201 & $1,69 \mathrm{c}$ & $16,75 \mathrm{a}$ & $16,50 \mathrm{ab}$ & $0,012 \mathrm{~cd}$ & $0,015 \mathrm{abc}$ & $0,018 \mathrm{abc}$ & $0,022 \mathrm{e}$ \\
\hline 400 & $1,33 \mathrm{ab}$ & $21,02 \mathrm{~cd}$ & $19,84 \mathrm{c}$ & $0,018 \mathrm{e}$ & $0,022 \mathrm{c}$ & $0,028 \mathrm{~cd}$ & $0,038 \mathrm{f}$ \\
\hline 796 & $1,99 \mathrm{~d}$ & $23,04 d$ & $22,00 \mathrm{~d}$ & $0,033 \mathrm{f}$ & $0,042 \mathrm{~d}$ & $0,043 \mathrm{~d}$ & $0,059 \mathrm{~g}$ \\
\hline
\end{tabular}

${ }^{(1)}$ Nas colunas, médias seguidas pela mesma letra não diferem entre si pelo teste de Tukey a $5 \%$ de probabilidade. ${ }^{(2)}$ Valores em mg de $\mathrm{N}$ orgânico $\mathrm{kg}^{-1}$ solo.

à adição de lodos. Com a metodologia utilizada, os teores iniciais de $\mathrm{N}$ total e de $\mathrm{C}$ orgânico nos tratamentos foram indicadores pouco precisos das doses de lodo aplicadas ao solo. Aos 63 dias de incubação, os teores de C orgânico foram mais elevados no solo dos tratamentos com aplicação de doses mais elevadas de lodo de esgoto, em relação aos teores no solo da testemunha (Tabela 3). Aumentos de C no solo com aplicação de lodo de esgoto também foram observados por Lindsay \& Logan (1998), em campo, com doses crescentes até $300 \mathrm{Mg} \mathrm{ha}^{-1}$. $\mathrm{O}$ aumento de $\mathrm{C}$ orgânico propicia melhorias em diversas propriedades do solo, e esta é uma das principais vantagens do uso de lodo de esgoto na agricultura, principalmente na recuperação de áreas degradadas. A aplicação de altas doses de lodo de esgoto ao solo, no entanto, pode liberar $\mathrm{N}$ mineral acima das necessidades de um cultivo, com risco potencial de lixiviação de nitrato no solo.

\section{Conclusões}

1. A fração de mineralização do N orgânico aplicado ao Latossolo via lodo, de Franca ou de Barueri, é estimada em $31 \%$.

2. A velocidade de mineralização do lodo de Franca independe das doses aplicadas, enquanto a do lodo de Barueri é diminuída nas doses elevadas.
3. Os lodos de esgoto acidificam o solo; a acidificação é mais acentuada com o lodo de Franca do que com o de Barueri.

\section{Referências}

AQUINO NETO, V. D. Avaliação do aproveitamento agrícola de lodos de curtume. 1998. 110 f. Dissertação (Mestrado em Solos e Nutrição de Plantas) - Escola Superior de Agricultura Luiz de Queiroz, Piracicaba, 1998.

BANERJEE, M. R.; BURTON, D. L.; DEPOE, S. Impact of sewage sludge application on soil biological characteristics. Agriculture, Ecosystems and Environment, Oxford, v. 66, n. 3, p. 241-249, 1997.

CAMARGO, F. A. O.; GIANELLO, C.; VIDOR, C. Potencial de mineralização do nitrogênio em solos do Rio Grande do Sul. Revista Brasileira de Ciência do Solo, Campinas, v. 21, n. 4, p. 575-579, 1997.

EPSTEIN, E.; KEANE, D. B.; MEISINGER, J. J.; LEGG, J. O. Mineralization of nitrogen from sewage sludge and sludge compost. Journal of Environmental Quality, Madison, v. 7, n. 2, p. 217-221, 1978.

GANGBAZO, G.; PESANT, A. R.; BARNETT, G. M.; CHARUEST, J. P.; CLUIS, D. Water contamination by ammonium nitrogen following the spreading of hog manure and mineral fertilizers. Journal of Environmental Quality, Madison, v. 24, n. 3, p. 420-425, 1995. 
GILMOUR, J. T.; SKINNER, V. Predicting plant available nitrogen in land-applied biosolids. Journal of Environmental Quality, Madison, v. 28, n. 6, p. 11221126, 1999.

LERCH, R. N.; AZARI, P.; BARBARICK, K. A.; SOMMERS, L. E.; WESTFALL, D. G. Sewage sludge proteins II: extract characterization. Journal of Environmental Quality, Madison, v. 22, n. 3, p. 625-629, 1993.

LINDEMANN, W. C.; CARDENAS, M. Nitrogen mineralization potential and nitrogen transformations of sludge-amended soil. Soil Science Society of America Journal, Madison, v. 48, n. 5, p. 1072-1077, 1984.

LINDSAY, B. J.; LOGAN, T. J. Field response of soil physical properties to sewage sludge. Journal of Environmental Quality, Madison, v. 27, n. 3, p. 534-542, 1998.

McCLUNG, G.; FRANKENBERGER, J. Soil nitrogen transformations as affected by salinity. Soil Science, Baltimore, v. 139, n. 6, p. 405-411, 1985.

PARKER, C. F.; SOMMERS, L. E. Mineralization of nitrogen in sewage sludges. Journal of Environmental Quality, Madison, v. 12, n. 1, p. 150-156, 1983.

PATRIQUIN, D. G.; BLAIKIE, H.; PATRIQUIN, M. J.; YANG, C. On-farm measurements of $\mathrm{pH}$, electrical conductivity, and nitrate in soil extracts for monitoring coupling and decoupling of nutrient cycles. Biological Agriculture and Horticulture, Bicester, v. 9, n. 2, p. 231272, 1993.

RAIJ, B. van; QUAGGIO, J. A. Métodos de análise de solo para fins de fertilidade. Campinas: Instituto Agronômico, 1983. 31 p. (Boletim Técnico, 81).

RODGERS, C. S.; ANDERSON, R. C. Plant growth inhibition by soluble salts in sewage sludge-amended mine spoils. Journal of Environmental Quality, Madison, v. 24 , n. 4 , p. 627-630, 1995.
ROWELL, D. M.; PRESCOTT, C. E.; PRESTON, C. M. Decomposition and nitrogen mineralization from biosolids and other organic materials: relationship with initial chemistry. Journal of Environmental Quality, Madison, v. 30, n. 4, p. 1401-1410, 2001.

RYAN, J. A.; KEENEY, D. R.; WALSH, L. M. Nitrogen transformations and availability of anaerobically digested sewage sludge in soil. Journal of Environmental Quality, Madison, v. 2, n. 2, p. 240-243, 1973.

SIKORA, L. J.; YAKOVCHENKO, V. Soil organic matter mineralization after compost amendment. Soil Science Society of America Journal, Madison, v. 60, n. 5, p. 14011404, 1996.

SILVA, C. A.; VALE, F. R.; ANDERSON, S. J.; KOBAL, A. R. Mineralização de nitrogênio e enxofre em solos brasileiros sob influência da calagem e fósforo. Pesquisa Agropecuária Brasileira, Brasília, v. 34, n. 9, p. 16791689, set. 1999.

SLOAN, J. J.; BASTA, N. T. Remediation of acid soils by using alkaline biosolids. Journal of Environmental Quality, Madison, v. 24, n. 6, p. 1097-1103, 1995.

SMITH, J. L.; SCHNABEL, R. R.; McNEAL, B. L.; CAMPBELL, G. S. Potential errors in the first-order model for estimating soil nitrogen mineralization potentials. Soil Science Society of America Journal, Madison, v. 44, n. 5 , p. 996-1000, 1980.

SMITH, S. R.; WOODS, V.; EVANS, T. D. Nitrate dynamics in biosolids-treated soils I: influence of biosolids type and soil type. Bioresource Technology, Amsterdam, v. 66, n. 2, p. 139-149, 1998.

STANFORD, G.; SMITH, S. J. Nitrogen mineralization potentials of soil. Soil Science Society of America Journal, Madison, v. 36, n. 2, p. 465-471, 1972.

TEDESCO, M. J.; GIANELLO, C.; BISSANI, C. A.; BOHNEN, H.; VOKWEISS, S. J. Análise de solo, plantas e outros materiais. 2. ed. Porto Alegre: UFRGS, 1995. (Boletim Técnico, 5). 